\title{
ON THE SPECIES OF BRACHININI (COLEOPTERA: CARABIDAE: BRACHININAE: BRACHININI) IN ROMANIAN MUSEUM COLLECTIONS
}

\author{
MELANIA STAN, PAUL GÎDEI
}

\begin{abstract}
The paper presents twelve Brachinini species from Romanian fauna. Brachinus nigricornis Gebler and B. brevicollis Motschulsky are firstly recorded in Romania. Distribution maps, based on the examined material, were made for each species. For seven Brachinus species, photos of the median lobe are given. Specimens preserved in the collections of "Grigore Antipa" National Museum of Natural History and Brukenthal National Museum were studied. Besides specimens collected from Romania, all the specimens/species which were sampled in other countries of Europe, Northern Africa and Indonesia (Java) are presented.
\end{abstract}

Résumé. Le travail présente douze espèces de Brachinini de la faune de Roumanie. Brachinus nigricornis Gebler et $B$. brevicollis Motschulsky sont enregistrées en Roumanie pour la première fois. Des cartes de distribution basée sur le matériel examiné ont été faites pour chaque espèce. Les photos du lobe médian sont données pour sept espèces de Brachinus. Les exemplaires conservés dans les collections du Muséum National d'Histoire Naturelle «Grigore Antipa» et le Muséum National Brukenthal ont été étudiés. En plus on présente des exemplaires collectés en Roumanie ainsi que des spécimens/espèces collectés dans d'autres pays d'Europe, d'Afrique du Nord et en Indonésie (Java).

Key words. Brachinus, Aptinus, Mastax, collections, Romania.

\section{INTRODUCTION}

In Romania, the Brachinini tribe is represented by three genera: Aptinus Bonelli, Brachinus Weber and Mastax Fischer von Waldheim. The species of the Brachinini tribe have been very poorly studied in Romania. There are old faunistical lists containing records of Brachinini, such as Bielz (1887), Fleck (1905), Montandon (1887, 1906), Jonesco (1911), Petri (1912, 1925-1926), Marcu (1929), Negru (1957), Săvulescu \& Popescu-Gorj (1964), Negru \& Roşca (1967), Ieniştea (1968, 1974, 1975). Besides these, there are few articles dealing with ecological and zoogeographical aspects, recording Brachinus explodens Dufts. and B. crepitans (L.) as the most frequent species in Romanian fauna. Recently, a checklist of Brachinini tribe, with seven species, was published by Nitzu (2007a).

\section{MATERIAL AND METHODS}

The paper presents data on the species/specimens preserved in the collections of the „Grigore Antipa” National Museum of Natural History, Bucharest and the Brukenthal National Museum, Sibiu. MGAB collections: Palaearctic Coleoptera collection, Dr. Nicolae Săvulescu collection and the collection organized by the corresponding author; the last collection is presented below as the examined material. BNM collections: Transylvanian Society collection, Karl Petri collection, Eugen Worell collection, Rolf Weyrauch collection and Eckbert Schneider collection. The specimens were sampled in Romania, Austria, Croatia, France, Germany, Greece, Italy, Kazakhstan, Portugal, Republic of Moldova, Spain, Ukraine, Algeria and Egypt. The collecting site is mentioned in part of the material, the rest having only generic information: 
Frankreich, Österreich, Siebenbürgen. The specimens were collected between 1858 (Transylvanian Society collection) and 1971 (Eckbert Schneider collection).

The identification was made on the basis of both outer and inner morphological features, using Hůrka's (1996) and Baehr's (2004) identification keys. All the specimens of Brachinus from both MGAB and BNM collections were studied/revised by the corresponding author. The specimens of Aptinus, Mastax and part of Brachinus crepitans and $B$. explodens of MGAB collections were identified by the coauthor.

The photos were taken using the stereomicroscope Stemi 2000-C and the digital camera Nikon D700, with Nikon 105 mm F2.8 lens.

Abbreviations:

Mt./Mts. - Mountain/s; spec. (s) - specimen/s; coll. - collection; MGAB - "Grigore Antipa" National Museum of Natural History; BNM - Brukenthal National Museum.

Abbreviations for the county names in Romania:

AB - Alba; AR - Arad; B - Bucureşti; BR - Brăila; BV - Braşov; BZ - Buzău; CJ - Cluj; CL - Călăraşi; CS - Caraş Severin; CT - Constanţa; DJ - Dolj; GL - Galaţi; GR - Giurgiu; HD - Hunedoara; IF - Ilfov; IL - Ialomiţa; MH - Mehedinţi; MM - Maramureş; MS - Mureş; OT - Olt; PH - Prahova; SB - Sibiu; SJ - Sălaj; SM - Satu Mare; SV - Suceava; TL - Tulcea; TR - Teleorman; VL - Vâlcea; VN - Vrancea; VS - Vaslui.

\section{RESULTS AND DISCUSSIONS}

In Romania, the genera Aptinus and Mastax are each represented by only one species, Aptinus bombarda Illiger, respectively Mastax thermarum thermarum (Steven). The genus Brachinus contains ten species. Twelve species of Brachinini collected from Romania were identified in the MGAB collections and six species of Brachinus from Romania were identified in the BNM collections.

The genera, subgenera and species are presented alphabetically. The classification of species in subgenera follows the site http://carabidae.org. The number of specimens, sex, the collecting sites alphabetically grouped by counties, the collecting data and the collector's name are mentioned for each species.

Besides the species present in Romanian fauna, in the MGAB collections there are several additional foreign species: Aptinus displosor (L. Dufour), A. pyrenaeus Dejean, Brachinus boeticus Rambur, B. italicus Dejean, B. pecoudi Puel, B. sclopeta (Fabricius), B. exhalans (P. Rossi), B. humeralis Ahrens, Pheropsophus africanus (Dejean) and P. javanus (Dejean). Additional foreign species are also present in BNM collections: Brachinus andalusiacus Rambur, B. boeticus Rambur, B. pygmaeus Dejean, B. bellicosus L. Dufour, B. italicus Dejean, B. sclopeta (Fabricius), B. exhalans (P. Rossi), B. humeralis Ahrens, B. quadriguttatus Gebler, B. lethierryi Reiche and $B$. oblongus (Dejean).

Genus Aptinus Bonelli, 1810

Aptinus bombarda Illiger, 1800

(Fig. $1 \mathrm{~A})$

Examined material: 1 spec., Băile Herculane, 14.07.1960, leg. N. Săvulescu; Moldova Nouă: 1 spec., 26.05.1971; 2 specs., 27.05.1971; 2 specs., 28.05.1971, leg. N. Săvulescu; Oraviţa (Marila): 2 specs., 15.07.1959; 1 spec., 1.07.1963; 3 specs., 1.07.1971; 1 spec., 2.07.1971; 1 spec., 3.07.1971, leg. N. Săvulescu (CS); 1 spec., Comana, 16.06.1955, leg. N. Săvulescu (GR); 9 specs., Periprava, 29.07.1967, leg. N. Săvulescu (TL).

Palaearctic collection: 1 ô, Buştenari, leg. R. Canisius (PH).

Records: Cluj-Napoca (Bielz, 1887), Bazna, Sighişoara (Petri, 1912), Cheile Turzii (Ruicănescu, 1992), Bârnova forest (Iaşi), (Varvara, 2004), Gîdinţ forest, 


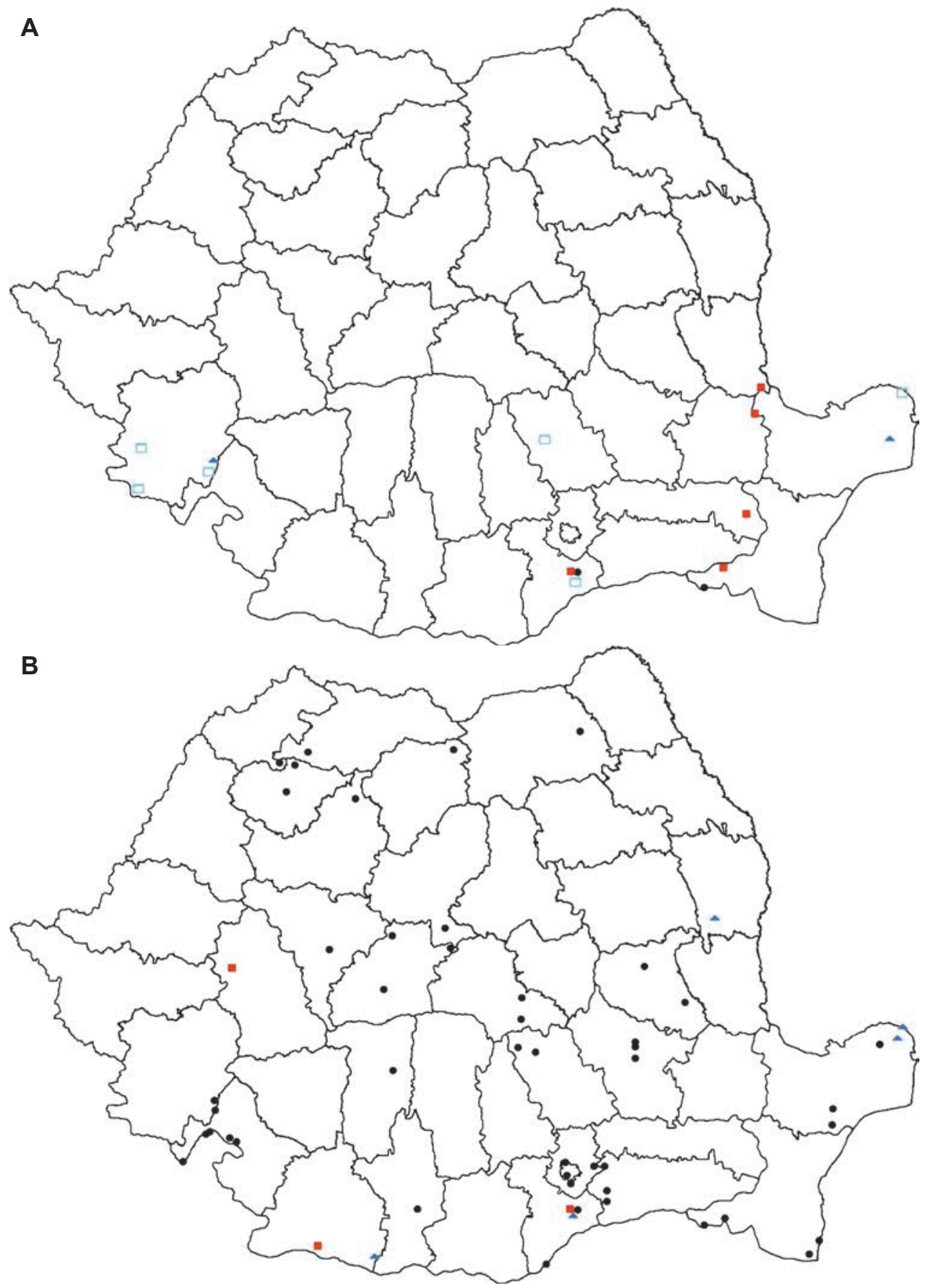

Fig. 1 - Distribution in Romania of Aptinus bombarda (empty blue squares), Brachinus bayardi (black bullets), B. bipustulatus (red squares), Mastax thermarum thermarum (bleu triangles) (A) and Brachinus crepitans (black bullets), B. ejaculans (red squares), B. plagiatus (bleu triangles) (B) based on examined material. 
Potoci (Neamţ) (Varvara \& Zugravu, 2006), Sic-Păstăraia (Nitzu, 2007b), Vaslui (Varvara, 2009).

Genus Brachinus Weber, 1801

Subgenus Brachinus Weber, 1801

Brachinus crepitans (Linnaeus, 1758)

(Figs. $1 \mathrm{~B} ; 4 \mathrm{~A}$ )

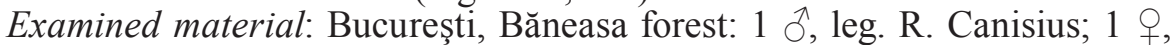

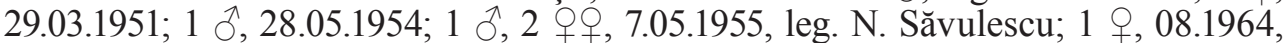
leg. A. Roşca; 1 đ̃, 20.09.1964, leg. Vl. Brădescu; 2 ơ, 1991, leg. Ş. Procheş (B); 1 गे, Braşov, $700 \mathrm{~m}$ asl, 20.06.1954, leg. I. Ceianu (BV); 1 今े, Cernătești, archaeological site, 31.08.2005, leg. D. Kurzeluk; Vizereanu hill (Beceni, Izvoru Dulce): 2 우,

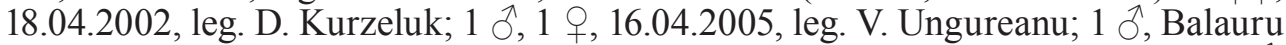
hill (on the west of Izvoru Dulce), 4.05.2005, leg. V. Ungureanu (BZ); Budeşti: 1 ô, 15.05.1974, leg. M. Weinberg; 1 O, 25.05.1979, leg. M. Weinberg; 1 9, 7.09.1979, leg. A. Dumitru; 1 đ̃, Vasilaţi, 18.05.1988, leg. R. Serafim; 1 क, Belciugatele, 13.07.2005, leg. V. Ungureanu (CL); Canaraua Fetii: 1 ô, 1 ㅇ, 29.06.1956; 1 spec., 16.05.1962, leg.

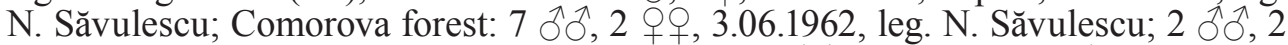

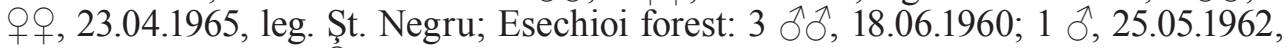
leg. N. Săvulescu; 1 + , Hagieni forest, 21-22.04.1965, leg. Şt. Negru (CT); Comana forest: 1 q, leg. A. L. Montandon; 1 +, 30.05.1954, leg. N. Săvulescu; 1 + 18.09 .1964 ,

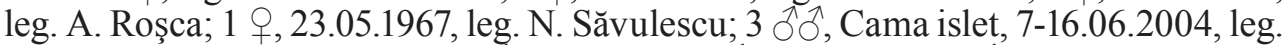

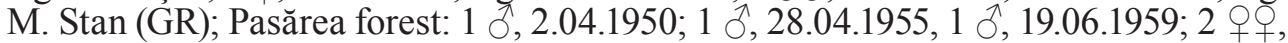
7.06.1960, leg. N. Săvulescu; 1 +, 18.09.1964, leg. A. Roşca; 1 \%, 23.05.1967, leg. N. Săvulescu (IF); 1 đ̂, Mireşu Mare, 06.1942, leg. E. Váradi (MM); 1 đ̂, Brezniţa-Ocol, 5.05.1962, leg. N. Săvulescu; Drobeta Turnu Severin: 1 , 12.07.1962; 1 , 1.08.1962, leg. N. Săvulescu; Eşelniţa: 1 q, 15.08.1965, leg. X. Scobiola Palade; 3 우, 10.08.1966 (under logs), leg. Şt. Negru; 1 क, 5.08.1967, leg. Şt. Negru; 1 \%, Orşova (Graţca), 30.03.1967 (under stone), leg. Şt. Negru; 1 O, Sviniţa, 28.03.1970, leg. N. Săvulescu $(\mathrm{MH}) ; 1$ ô, 1 P, Pestra, Drăgănești-Olt, 6.04.1980, leg. G. Andrei (OT); 1 \&, Valea

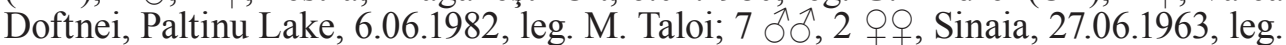
A. Roşca (PH); 1 ô, Sibiu, 29.06.1961, leg. I. Nemeș (SB); 1 O, Suceava, 15.07.1959,

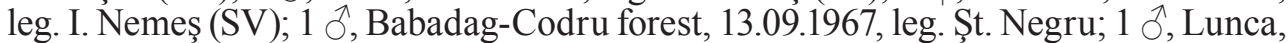
31.05.1977, leg. G. Andrei; 1 , Maliuc, mila 26, 7.08.1991, leg. R. Serafim (TL); 1 , Olănești, 9.09.1982, leg. A. Popescu-Gorj (VL); Răstoaca, Putna: 2 우, 9.09.1975; 1 ô, 1 o, 13.09.1975, leg. N. Săvulescu; 1 ô, Vidra, 23.07.1962, leg. A. Roşca (VN); 1 O’, 1 O, Ineu Mt. (Rodna Mts), 28.06.1955, leg. E. Schneider. Palaearctic collection: București: 1 ㅇ, leg. R. Canisius; 1 ð, leg. A. L. Montandon, named Brachinus psophia Serv. (B); 1 Oे, Jilava, leg. A. L. Montandon (IF); 1 q, Besançon, France, coll. A. L. Montandon.

Transylvanian Society collection: 1 ô, 1 ㅇ, Banat, Birthler, 1895; Siebenbürgen (Transylvania): 2 ふึત, Birthler, 1895; 1 ㅇ, Kronstädter Gbg., leg. F. Deubel (BV);

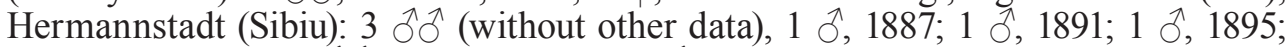

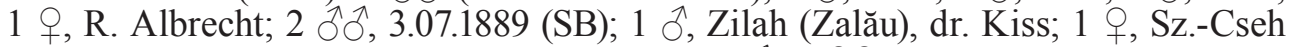
(Szilágycseh) (Cehu Silvaniei), dr. Kiss (SJ); 1 , 3 우, Hadad (Hodod), dr. Kiss

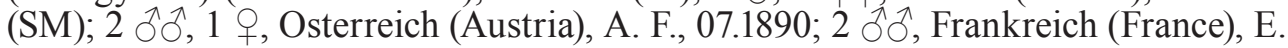
A. Bielz, 1858 (named B. psophia Serv. and B. explodens var. glabratus Dej).

Karl Petri collection: $1 \hat{\jmath}$, Dees (Dej), 1890, leg. K. Petri (CJ); Schassburg (Sighişoara): 1 đ̂, 1 \%, 1884, leg. K. Petri; 1 q, 05.1899, leg. K. Petri (MS); 1 \%, Baassen (Bazna), 07.1909 (SB). 


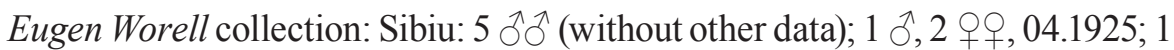
, Karlsburg (Alba Iulia), named Brachinus ganglbaueri Afb. (AB); Chişinău (Republic

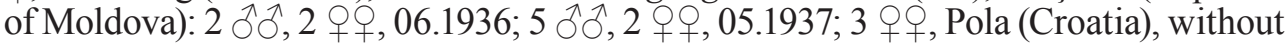
other data, named Brachinus immaculicornis Dej.

Rolf Weyrauch collection: Herkulesbad (Băile Herculane): 1 , 19.05.1956; 1

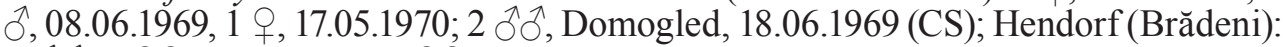
2 đō, 4 우, 23.05.1954; 3 우, 15.05.1955, leg. R. Weyrauch (SB).

Records: Mangalia (Montandon, 1887), Agnita, Bistriţa, Braşov, Cincu, ClujNapoca, Dej, Deva, Făgăraş, Geaca, Haţeg, Mediaş, Prejmer, Reghin, Săcele, Sibiu, Sighişoara (Bielz, 1887), Iglița, Mangalia (Fleck, 1905), Comana (Montandon, 1906), Iaşi (Jonesco, 1911), Bahna (Marcu, 1929), Comorova (Negru, 1957, Negru \& Roşca, 1967), Ieşelniţa valley (Ieniştea, 1975), Hagieni (Negru \& Roşca, 1967, Nitzu, 2001), Singureni (Fiera et al., 2013).

\section{Brachinus ejaculans Fischer von Waldheim, 1828}

(Figs. 1 B; 4 C, D)

Examined material: 1 ,, Bistret, 23-25.06.1981, leg. D. Dumitrescu(DJ); Comana:

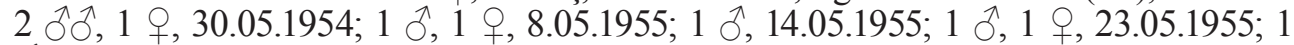
ô, 3.06.1955, leg. N. Săvulescu (GR); 1 + , Dobra, 20.05.1970, leg. I. Ceianu (HD).

Karl Petri collection: 1 O, 1 \%, Sebastopol, Krim (Crimea), 8.05.1906, leg. \& det. W. Pliginskyi.

Records: Nitzu, 2007a (without collecting data).

\section{Brachinus elegans Chaudoir, 1842}

(Figs. 2 A; 5 A)

Examined material: Herăstrău Park, București: 1 + , 30.05.1991, leg. H. Tănase; 1 q, 14.06.1991, leg. H. Tănase (B); 2 đ̂̀ ô, Budeşti, 25.05.1979, leg. M. Weinberg; 1 q, Ceianu Nou islet, km 342 on the Danube River, 26.05.2008, leg. M. Stan (CL); 1 o, Băile Herculane, 28.06.1958, leg. N. Săvulescu (CS); 1 \&, Canaraua Fetii, 29.06.1956, leg. N. Săvulescu (CT); 1 đે, Bistreț, 25.06.1981, leg. R. Serafim; Panaghia forest, Radovan: 1 đ̂, 14.06.1984, leg. G. Andrei; 1 ô, 15.06.1984, leg. M. Andrei; 1 ô, Gătanu islet, 16.05.2004, leg. O. Popa (DJ); Comana: 1 क, 30.05.1954, leg. N. Săvulescu; 3 ổ, 1

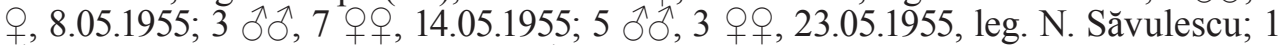
ते, 3.06.1955, leg. N. Săvulescu; 1 ô, 18.09.1964, leg. A. Roşca; 1 + 9.06.1965, leg. N. Săvulescu; 1 o, 28.03.1977, leg. G. Andrei; 1 ô, 1 क, Cama islet, km 510 on the Danube River, 7-16.06.2004, leg. M. Stan (GR); 1 o, Mogoşoaia, 27.06.1961, leg. A. Roşca; 1 ô, Pasărea forest, 3.06.2001, leg. C. Pârvu (IF); 2 ợ, Jupalnic, 20.04.1968,

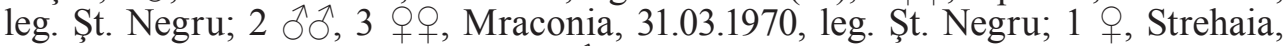
10.05.1982, leg. R. Serafim (MH); 1 ô, Sighetu Marmaţiei, 23.07.1953, leg.? (MM); 1 , Palanca forest, Nicolae Titulescu, 23.06.1980, leg. I. Matache (OT); 1 ô, Lunca, 31.05.1977, leg. G. Andrei (TL); 1 ð, Marburg, Hessen (Germany), coll. I. Ceianu; 1 ${ }^{\lambda}$, Gallia, Reitter, coll. I. Ceianu.

Palaearctic collection: 1 đે, Also-Rákos (Racoşul de Jos), coll. F. Deubel, named Brachinus crepitans (L.) (BV); 1 đ, 2 qo+, Dordogne, France, coll. A. L. Montandon, named B. psophia Serv.

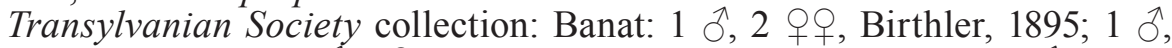

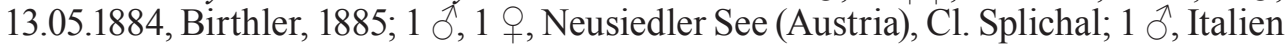
(Italy), E. A. Bielz, 1858 (all the specimens as B. psophia Serv.)

Karl Petri collection: Schassburg (Sighişoara): 1 ô, 1884 (named Brachinus psophia Serv.); 1 đ, 1 ㅇ, 1889; 3 우, 1890 (named B. psophia Serv.); 1 ㅇ, 1891 (MS); 

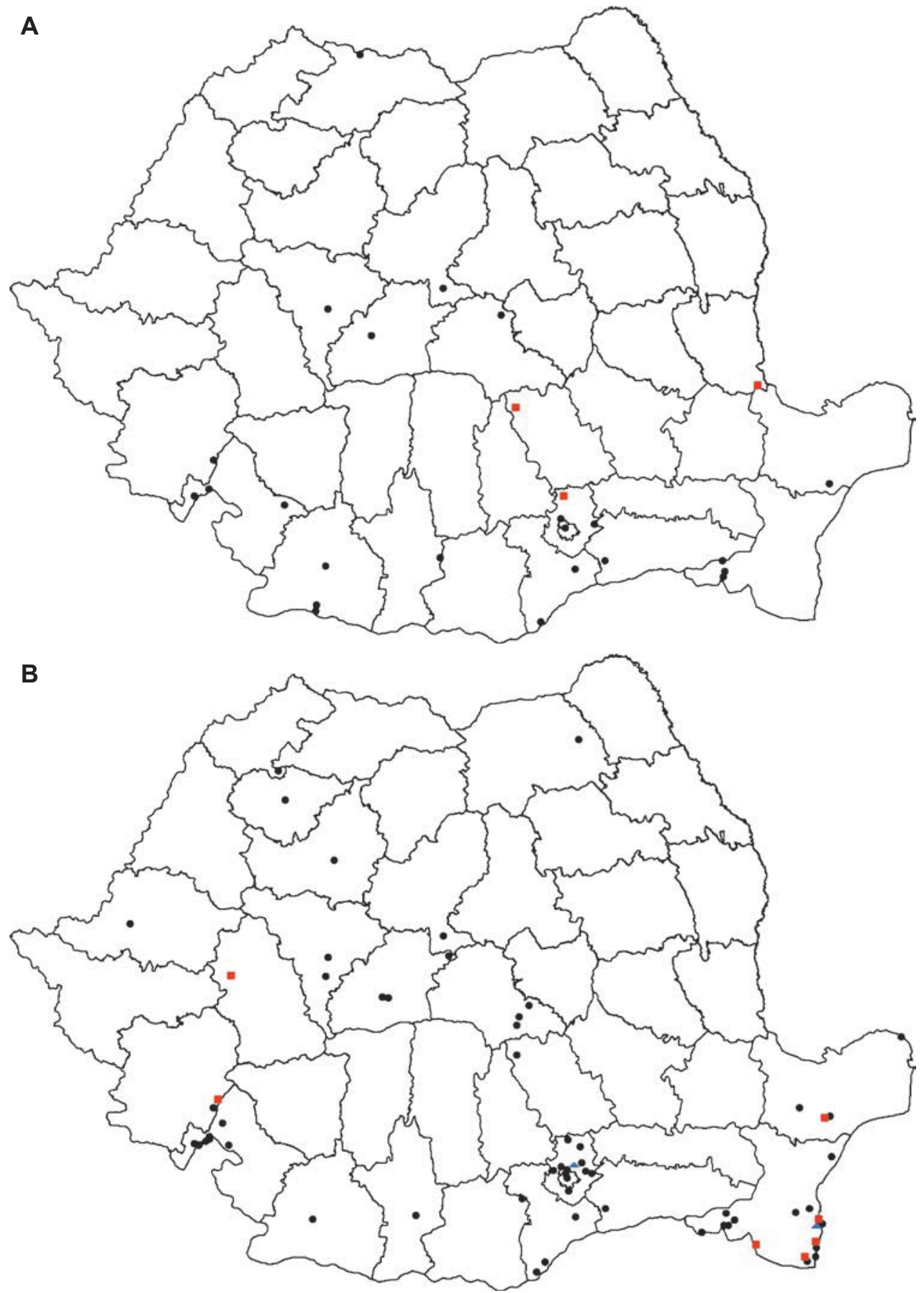

Fig. 2 - Distribution in Romania of Brachinus elegans (black bullets), B. psophia (red squares) (A) and $B$. brevicollis (red squares), B. explodens (black bullets), B. nigricornis (blue triangles) (B) based on examined material. 


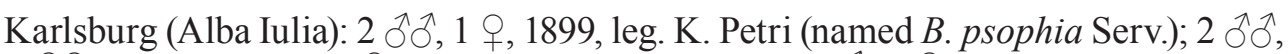
2 우, 07.1912 (AB); 1 , Neusiedler See (Austria); 1 ภ, 1 ㅇ, Marburg (Germany), Weber; 1 ô, Sebastopol, Krim (Crimea), 29.07.1901, leg. W. Pliginskyi.

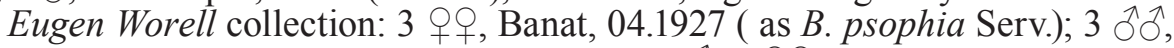
2 우, Carmen Sylva (Eforie Sud), 06.1931 (CT); 1 ภิ, 3 우, Chişinău (Republic of Moldova), 05.1937, as Brachinus crepitans (L.); 2 우, Neusiedler See (Austria), as B. psophia Serv.; 1 ô, Marburg (Germany), Weber.

Rolf Weyrauch collection: Canaraua Fetii: 1 ภ, 22.06.1974, 1 ภ, 3 우, 1518.05.1975, named Brachinus crepitans (L.) (CT); 1 ㅇ, Salzburg (Ocna Sibiului), 5.06.1956, named B. explodens Dufts., leg. R. Weyrauch (SB).

Eckbert Schneider collection: 1 + Iortmac valley, 25-27.04.1971, leg. E. Schneider, named B. psophia Serv. (CT).

Records: Alba Iulia, Sighişoara (Petri, 1925-1926), Mraconia valley (Ieniştea, 1975), Cheile Turzii (Ruicănescu, 1992), Casian, Cheia, Gura Dobrogei cave (Nitzu, 2001). All records are named Brachinus ganglbaueri Afb.

\section{Brachinus plagiatus Reiche, 1868}

(Figs. 1 B; 4 B)

Examined material: Romania: 1 đ, Dăbuleni, 5.06.1986, leg. I. Ceianu (DJ); Comana: 1 đ, 30.05.1954; 1 đ, 1 क , 23.05.1955; 1 क, 3.06.1955, leg. N. Săvulescu; 1

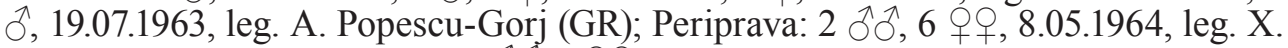
Scobiola (det. M. Al. Ieniştea); 2 ô ô, 8 우, 9.05.1964, leg. M. Weinberg \& X. Scobiola (det. A. Roşca, Şt. Negru, M. Al. Ieniştea); 1 o, 19.04.1966, leg. Şt. Negru (det. M. Al. Ieniştea); 1 + , Letea forest, 20.07.1966, leg. Şt. Negru (det. M. Al. Ieniştea) (TL);

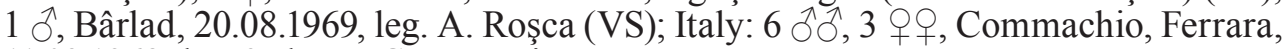
11.03.1968, leg \& det. P. Garagnani.

Transylvanian Society collection: 2 specs., Frankreich (France), E. A. Bielz, 1858.

Karl Petri collection: Sebastopol, Krim (Crimea): 1 , , 07.03.1906, 1 , 20.02.1908, 1 ㅇ (without other data), leg. \& det. W. Pliginskyi.

Eugen Worell collection: 10 , Cartagena (Spain).

Records: Letea forest, Periprava (Ieniştea, 1968).

\section{Brachinus psophia Audinet-Serville, 1821}

(Figs. 2 A; 5 B)

Examined material: 1 đ̄, Brateş Lake, 2.06.1952, leg. N. Săvulescu (GL); 1 q, Cocioc, 11.05.1991, leg.? (IF); 2 §ోَ, Sinaia, 27.06.1963, leg. A. Roşca (PH).

Records: Dej, Deva, Dobra, Hațeg, Mediaș (Bielz, 1887), Măcin (Fleck, 1905), București, Comana (Montandon, 1906), Maliuc, Mila 26 (Popovici, 1992), Sic-Păstăraia (Nitzu, 2007b).

Subgenus Brachynolomus Reitter, 1919

Brachinus brevicollis Motschulsky, 1844

(Figs. 2 B; 3 A; 5 D)

Examined material: Romania: 1 \&, Băile Herculane, 15.06.1952, leg. N. Săvulescu (CS); Agigea: 1 ô, 1 क, 24.04.1963, leg. Şt. Negru; 1 +, 30.08.1963, leg.

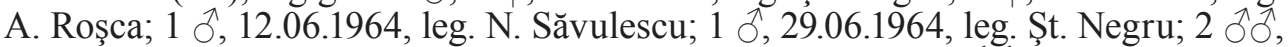

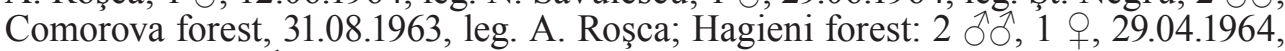
leg. Şt. Negru; 1 đૈ, 9.05.1965, leg. N. Săvulescu; 1 †, Dumbrăveni, 23.05.1963, leg. N. Săvulescu (CT); 1 +, Dobra, 20.05.1970, leg. I. Ceianu (HD); Babadag-Codru forest: 

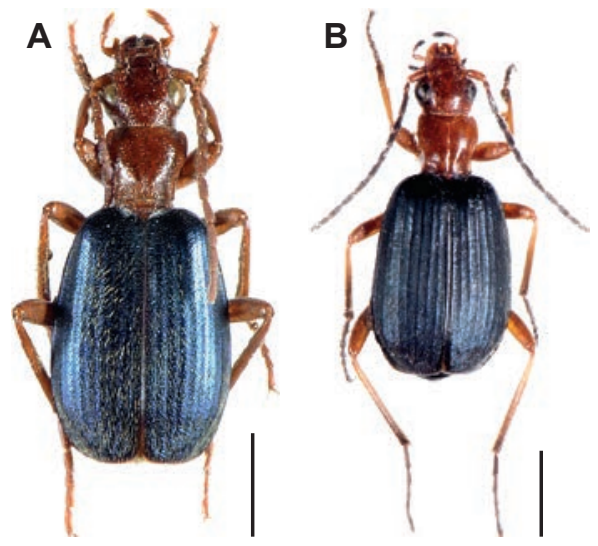

Fig. 3 - Habitus: Brachinus brevicollis Motschulsky, male (A) and Brachinus nigricornis Gebler, female (B). Scale: 2 mm. (photos: G. Năzăreanu)
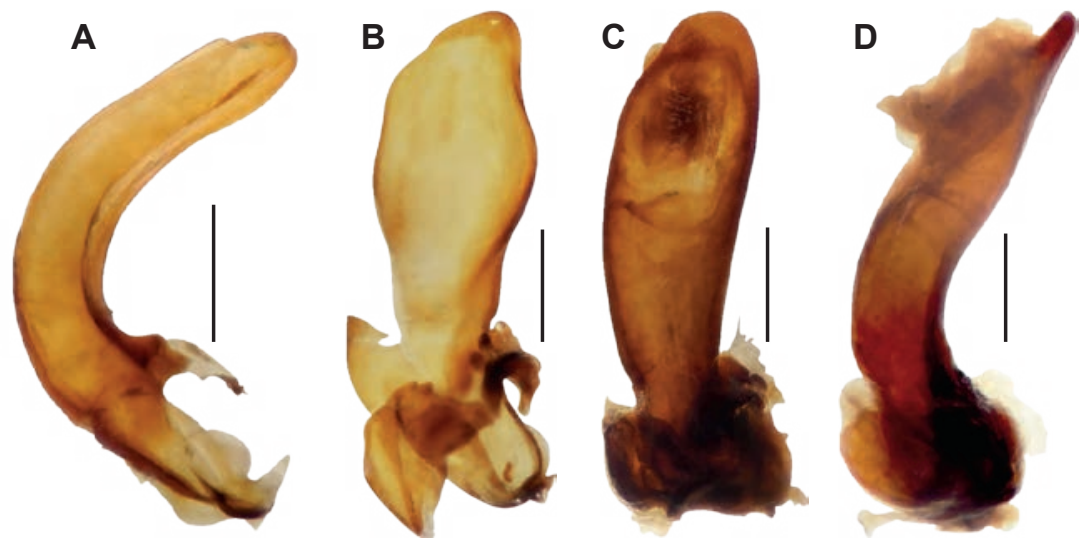

Fig. 4 - Median lobe: Brachinus crepitans (lateral view) (A), B. plagiatus (ventral view) (B), B. ejaculans (ventral and lateral views) $(C, D)$. Scale: $1 \mathrm{~mm}$. (photos: G. Năzăreanu)
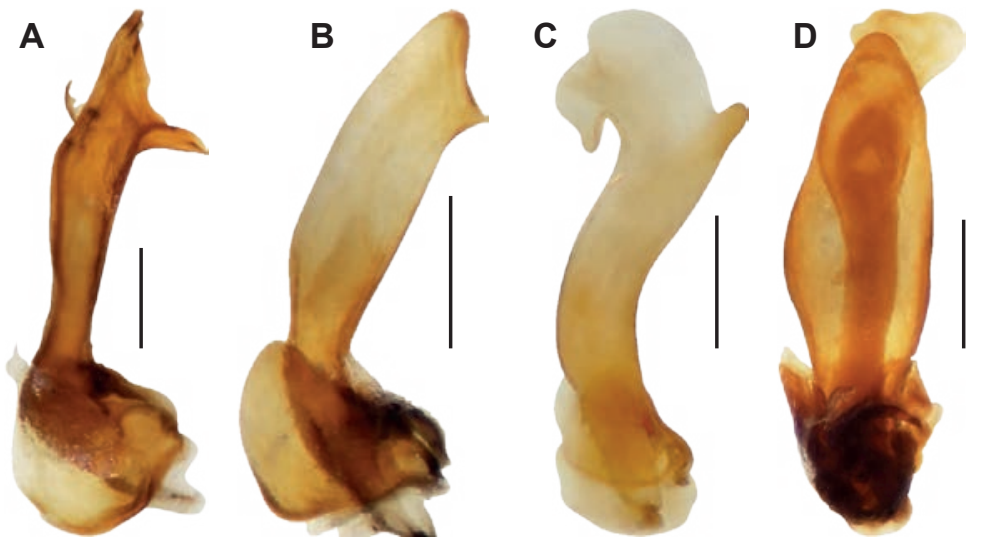

Fig. 5 - Median lobe: Brachinus elegans (lateral view) (A), B. psophia (lateral view) (B), B. explodens (lateral view) (C), B. brevicollis (ventral view) (D). Scale: 1 mm. (photos: G. Năzăreanu) 


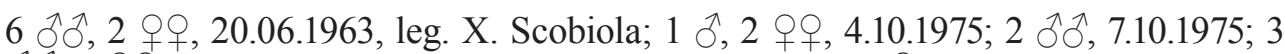

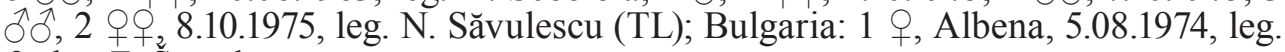
\& det. Z. Sustek.

Remarks: New record for Romania.

Brachinus explodens Duftschmid, 1812

(Figs. 2 B; 5 C)

Examined material: 1 ô,, Şiria, 11.07.1954, leg.? (AR); Bucureşti: 2 ôे, Băneasa, 29.03.1951, leg. N. Săvulescu; 1 ô, Botanical Garden, 5.05.1991, leg. H. Tănase; Herăstrău Park: 1 \%, 10.05.1991; 1 +, 5.06.1991, leg. H. Tănase; 1 q, 1991, leg. Ş. Procheş (B); Budeşti: 1 đ̃, 15.05.1958, leg. A. Roşca; 1 ô, 25.05.1979, leg. G. Andrei; 8 specs., 7.06.1979, leg. A. Dumitru (CL); 1 spec., Băile Herculane, 15.06.1952, leg. N. Săvulescu (CS); 1 , , Băneasa forest, 15.07.1961, leg. N. Săvulescu; Canaraua Fetii: 2 우, 29.06.1956, leg. N. Săvulescu; 3 ổ, 15.07.1961, leg. N. Săvulescu; 1 spec., 1.05.1965, leg. X. Scobiola; 1 ㅇ, 1.10.1965, leg. Șt. Negru; Comorova forest: 1

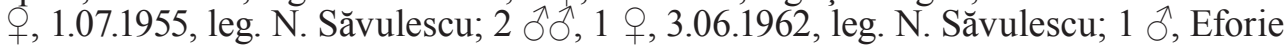
Sud, 22.06.1952, leg. N. Săvulescu; Esechioi: 3 우, 18.06.1960; 1 + , 25.05.1962, leg. N. Săvulescu; Hagieni forest: 1 spec., 29.04.1964, leg. Şt. Negru; 1 Oૈ, 25.09.1965, leg. Şt. Negru; 12 specs., Murfatlar, 8.10.1951, leg. N. Săvulescu; 1 \&, Negureni forest, 20.05.1994, leg. G. Andrei; 1 +, Oltina forest, 13.05.1958, leg. N. Săvulescu; Valu lui Traian: 1 o, 19.10.1953; 2 우, 25.10.1953, leg. X. Scobiola (CT); Perişor forest:

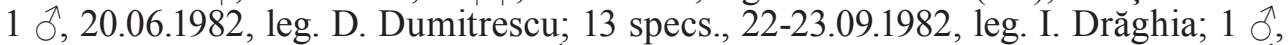

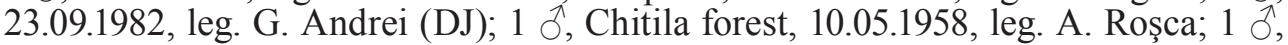
Moara Domnească, 9.07.2008, leg. M. Stan; Mogoşoaia: 1 +, 15.04.1954, leg. X. Scobiola; 1 q, 4.07.1957, leg. I. Sienkiewicz; 1 \% 9.05.1959, leg. V. Iuga; Pasărea forest: 5 specs., 28.04.1955, leg. N. Săvulescu; 1 O, 7.06.1959; 1 , 18.05.1967, leg. N. Săvulescu; 1 Ò, Snagov, 25.04.1957, leg. A. Roşca; 1 Ò, Ştefăneşti, 31.10.1953,

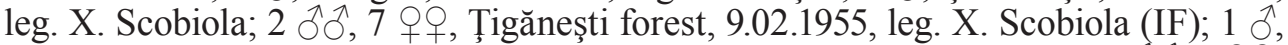
Cetăţuia, km 516 on the dike, 26.04.2004, leg. C. Pârvu; Comana forest: 4 đô, 5 o Oq,

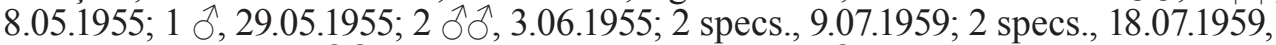
leg. N. Săvulescu; 3 우, 18.09.1964, leg. A. Roşca; 1 , Malu, km 508 on the dike, 27-29.04.2004, leg. M. Stan (GR); 1 q, Brezniţa-Ocol, 5.05.1962, leg. N. Săvulescu; 1 q, Cireşu, 7.07.1965, leg. N. Săvulescu; 3 specs., Eşelniţa, 12.08 .1966 (under logs), leg. SSt. Negru; 4 specs., Jupalnic, 28.03.1967 (under stones), leg. Şt. Negru; 1 O, 4 우, Mraconia, 31.03.1970, leg. Şt. Negru; 1 J, 2 우, Ogradena, 5.06.1968, leg. X. Scobiola; Orşova: 30 specs., 30.03.1967 (under stones); 1 + , 27.04.1968; 1 ㅇ, 3.04.1969, leg. Şt. Negru; Strehaia: 1 q, 10.05.1982, leg. R. Serafim; 1 + , 16.05.1982, leg. D. Dumitrescu (MH); Pestra, Drăgăneşti-Olt: 1 spec., 4.04.1980; 1 spec., 6.04.1980, leg. G. Andrei (OT); 1 ㅇ, Sinaia, 27.06.1963, leg. A. Roşca (PH); 1 ò, Suceava, 23.05.1959, leg. I. Nemeş (SV); Babadag-Codru forest: 1 spec., 20.06.1958, leg. N. Săvulescu; 5 specs., 14.09.1967, leg. Şt. Negru; 9 specs., 15.09.1967, leg. Şt. Negru; 1 q, Periprava, 4.08.1965, leg. SSt. Negru (TL); 3 우, Zăvestreni forest, 20.06.1978, leg. R. Serafim (TR); 1 spec., Corfu, Greece, leg. A. Hetschko, coll. I. Ceianu; 1 +, Majevica Mts, Bosnia \& Herzegovina, leg. Vl. Zoufal, coll. I. Ceianu.

Palaearctic collection: 1 ○, Bucureşti, leg. R. Canisius (B); 1 q, Honigberg (Hărman), coll. Fr. Deubel; 1 +, Braşov Mts, coll. Fr. Deubel (BV); 1 Ò, Mangalia,

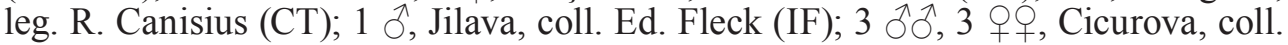
Ed. Fleck (TL).

Transylvanian Society collection: 1 +, Kapellenberg (Tâmpa Mt.), leg. F. Deubel (BV); Banat: 1 ㅇ, 23.04.1885, Birthler, 1895; 1 ภ, 2 우, Birthler, 1895; 2 
${ }_{+}$, Schassburg (Sighişoara), 1889, leg. K. Petri (MS); Hermannstadt (Sibiu): 1 q,

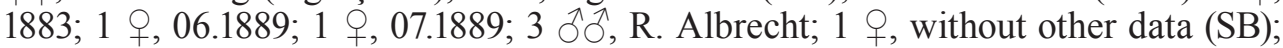
1 ग, 1 , Zilah (Zalău), dr. Kiss (SJ); 1 ô, 2 우, Hadad (Hodod), dr. Kiss (SM); 1

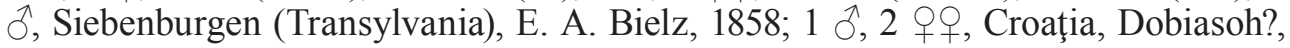
Birthler, 1895.

Karl Petri collection: Karslburg (Alba Iulia): 1 , 1893; 1 đ̃, 4 우, 07.1907, leg. K. Petri (AB); 1 q, Klausenburg (Cluj Napoca), leg. Marrloff (CJ); Schassburg (Sighişoara): 1 ô, 4 우 (without other data); 1 q, 1884, leg. K. Petri; 2 우, 1889,

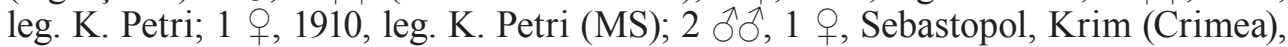
06.04.1908, leg. \& det. W. Pliginskyi; 1 ふै, Dalmatien, Spalato (Croatia), 1894, leg.?

Eugen Worell collection: 2 ờ, Băile Herculane, 06.1949 (CS); 2 우, Carmen

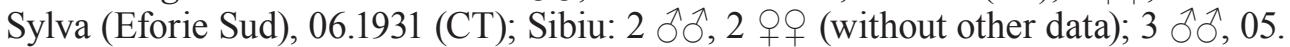
1932, E. Worell; Neppendorf (Turnişor): 1 †, 31.03.1948; 2 ふ઼َ, 28.04.1948 (SB);

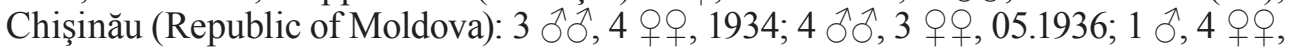
Pola (Croatia).

Rolf Weyrauch collection: 3 우, Petersdorf (Petreşti), 10.04.1955 (AB); 1 o, 1 q, Hendorf (Brădeni), 23.05.1954; 1 ふे, Bungard, 1.06.1969 (SB).

Eckbert Schneider collection: 1 q, Histria, 1-5.05.1971, leg. E. Schneider (CT).

Records: Braşov, Cătina, Cincu, Dej, Geaca, Haţeg, Mediaş, Prejmer, Reghin, Sibiu, Sighişoara (Bielz, 1887), Babadag, Bucureşti, Ciucurova (Fleck, 1905), Mangalia (Montandon, 1887, 1906, Nitzu, 2001), Iaşi (Jonesco, 1911), Alba Iulia, Cluj-Napoca, Hărman, Sighişoara (Petri, 1912), Comorova, Hagieni (Negru, 1957, Negru \& Roşca, 1967), Orşova, Mraconia valley (Ienistea, 1975), Movile karstic area (Nitzu, 1997), Agighiol, Babadag, Canaraua Fetei (Nitzu, 2001).

\section{Brachinus nigricornis Gebler, 1829}

(Figs. 2 B; 3 B)

(B).

Examined material: 1 q, Bucureşti, Băneasa forest, 7.05.1955, leg. N. Săvulescu

Transylvanian Society collection: 1 q, Algier, Birthler, 1895; 1 q, Frankreich (France), E. A. Bielz, 1858.

Eugen Worell collection: 1 ㅇ, Carmen Sylva (Eforie Sud), 06.1931 (CT); 1 , Algier (Algeria), without other data.

Karl Petri collection: 2 9 , Gallia, Béziers (France); 1, Russia mer., Uralsk (Oral, Kazakhstan).

Remarks: This is a new record for Romanian fauna.

Subgenus Cnecostolus Reitter, 1919

Brachinus bayardi Dejean, 1831

(Fig. $1 \mathrm{~A})$

Examined material: 1 \%, Esechioi, 18.06.1960, leg. N. Săvulescu (CT); Comana

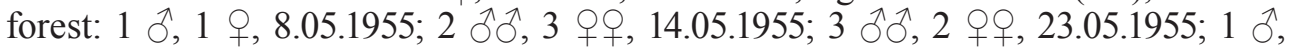
3.06.1955; 1 , 12.05.1965, leg. N. Săvulescu (GR).

Nicolae Săvulescu collection: Comana forest: 1 ๆ, 8.05.1955; 1 ô, 14.05.1955; 2 우, 3.06.1955, leg. N. Săvulescu (GR).

Records: Comana (Montandon, 1906, Săvulescu \& Popescu-Gorj, 1964), Esechioi (Săvulescu \& Popescu-Gorj, 1964). 
Brachinus bipustulatus Quensel, 1806

(Fig. 1 A)

Examined material: 1 Ô, 2 우, Smârdanul Nou, 29.05.1955, leg. N. Săvulescu (BR); 4 specs., Oltina Lake, 27.07.1954, leg. N. Săvulescu (CT); 1 +, Brateş Lake,

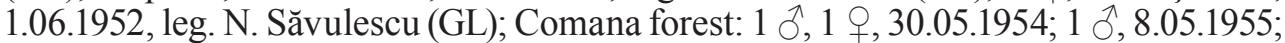

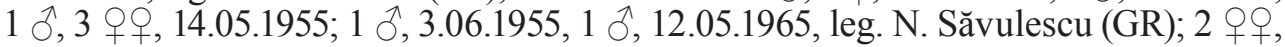
Saltava Lake, 20.06.1965, leg. N. Săvulescu (IL).

Palaearctic collection: 2 우, Comana, coll. A. L. Montandon (GR).

Transylvanian Society collection: 1 $\hat{\jmath}, 1$ ㅇ, Banat, 22.04.1885, Birthler.

Karl Petri collection: 1 , Russia mer., Uralsk (Oral, Kazakhstan)

Eugen Worell collection: 2 우, Attica (Greece), coll. Ed. Reitter; 1 , Chişinău (Republic of Moldova), 06.1936.

Records: Mangalia, Comana (Montandon, 1906).

Genus Mastax Fischer von Waldheim, 1828

Mastax thermarum thermarum (Steven, 1806)

(Fig. $1 \mathrm{~A})$

Examined material: 1 spec., Băile Herculane, 27.05.1982, leg. Nicolae Săvulescu (CS); Caraorman: 21 specs., 6.07.1968, leg. Şt. Negru \& A. P. Gorj \& N. Săvulescu (det. M. Al. Ieniştea, Şt. Negru); 56 specs., 3.10.1968; 27 specs., 4.10.1968; 1 spec., 7.10.1968; 11 specs., 4.10.1969, leg. X. Scobiola; 3 specs., 25.06.1991, leg. G. Andrei (TL).

Records: Caraorman, surroundings of Siutghiol Lake (Ieniştea, 1974).

Taking into account the examined material, we estimate that Brachinus psophia Serv. occurs more rarely than $B$. elegans Chaud., the majority of specimens identified as $B$. psophia belonging in fact to $B$. elegans. Brachinus plagiatus Reiche recorded from Periprava, Letea forest, 1966 was published by Ieniştea, in 1968. Brachinus brevicollis Motsch., a new record for Romania, is known from neighboring countries such as: Bulgaria, Republic of Moldova and Ukraine. Brachinus nigricornis Gebler, also a new record for Romanian fauna, is known only in south and southeastern Romania. Regarding the BNM collections, we conclude that the specimens identified as Brachinus crepitans (L.) collected from Canaraua Fetii (southeastern Romania) are actually Brachinus elegans Chaud., all the specimens identified as Brachinus psophia Serv. are Brachinus elegans Chaud. and the specimens identified as Brachinus immaculicornis Dej. (Pola/Croatia and Banat/Romania, 1895) are Brachinus crepitans (L.)

Other Brachinini species preserved in the MGAB and BNM collections (not present in Romanian fauna)

We used the site carabidae.org for the classification into subgenera.

Aptinus (Aptinidius) displosor (L. Dufour, 1811)

Palaearctic collection (MGAB): 2 $\widehat{\partial}$, Pyrénées-Orientales (France), coll. A. L. Montandon.

Aptinus (Aptinus) pyrenaeus Dejean, 1825

Palaearctic collection (MGAB): 3 $\hat{\delta} \hat{\sigma}$, Bagnères-de-Luchon (France), coll. A. L. Montandon (det. Leseleuc, 1878); 2 우, Pyrénées-Orientales (France), coll. A. L. Montandon. 
Brachinus (Brachinoaptinus) andalusiacus Rambur, 1837

Transylvanian Society collection (BNM): 1 , Spain, E. A. Bielz, 1858.

Brachinus (Brachinoaptinus) bellicosus L. Dufour, 1820

Karl Petri collection (BNM): 1 ô, Portugal (without other data).

Brachinus (Brachinoaptinus) boeticus Rambur, 1837

Nicolae Săvulescu collection (MGAB): 1 §̋, 3 우, Albergo de la Universidad, Sierra Nevada (Spain), leg \& det. H. Franz.

Karl Petri collection (BNM): 1 đ̄, 2 우, Andalusia, P. Plateria (Spain).

Eugen Worell collection(BNM): 2 ふैगे, Andalusia, P. Plateria, 27.06.1895 (Spain).

Brachinus (Brachinoaptinus) italicus Dejean, 1831

Palaearctic collection (MGAB): 1 ๙ , 1 ㅇ, Ponte Galeria, Roma (Italy), 2.04.1975, leg. \& det. S. Bruschi.

Karl Petri collection (BNM): 2 ふ઼े, Rome (Italy) (without other data).

Brachinus (Brachinoaptinus) lethierryi Reiche, 1868

Karl Petri collection (BNM): 1 Ỏ, 1 ๆ, Bône (Annaba, Algeria).

Brachinus (Brachinoaptinus) pecoudi Puel, 1925

Nicolae Săvulescu collection (MGAB): 2 9 +, Picos de Europa Mt., surroundings of Peña Vieja (Spain), leg. \& det. H. Franz.

Brachinus (Brachinoaptinus) pygmaeus Dejean, 1826 Schramm.

Karl Petri collection (BNM): 1 ô, 1 क, Tarifa, Andalusia (Spain), leg. G.

Eugen Worell collection (BNM): 1 đ̊, Tarifa, Andalusia(Spain), leg. G. Schramm.

Brachinus (Brachynolomus) oblongus Dejean, 1825

Karl Petri collection (BNM): 1 ō, 1 \%, Alexandria (Egypt).

Brachinus (Brachynolomus) sclopeta (Fabricius, 1792)

Palaearctic collection (MGAB): 3 specs., Brioni [Brijuni] (Croatia), leg. R. Canisius; 1 spec., Meine-Loire (France), leg. A. L. Montandon; 1 , Meine-Loire (France), coll. A. L. Montandon (named Brachinus bombarda Dej.).

Transylvanian Society collection (BNM): 3 우, France, E. A. Bielz, 1858; 1 , Gallia, Merk1.

Karl Petri collection (BNM): 2 ふ઼َ, Gallia merid. (without other data).

Eugen Worell collection (BNM): 30 specimens, without collecting data.

Brachinus (Cnecostolus) exhalans (P. Rossi, 1792)

Palaearctic collection (MGAB): 3 9 , La Tour Carbonniere, Gard, SaintLaurent-d'Aigouze (France), 18.04.1962, leg. \& det. G.M.?

Transylvanian Society collection (BNM): 1 q, France, E. A. Bielz, 1858.

Karl Petri collection (BNM): 290 , Gallia, Bordeaux (France).

Eugen Worell collection (BNM): 1 गे, France, without other data.

Brachinus (Cnecostolus) quadriguttatus Gebler, 1829

Karl Petri collection (BNM): 1 q, Russia mer., leg. Kelecsenyi. 
Brachinus (Dysbrachinus) humeralis Ahrens, 1812

Palaearctic collection (MGAB): 2 specs., Pollensa, Mallorca (Spain), 19.05.1895, donated by Auguste Puton, det. A. Puton as Brachinus causticus Serv.

Transylvanian Society collection (BNM): 1 spec., France, E. A. Bielz, 1858.

Pheropsophus (Stenaptinus) africanus (Dejean, 1825)

Palaearctic collection (MGAB): 1 spec., Gafsa, 04.1873; 1 spec., Kairouan, 10.04.1873; 1 spec. Ludien, 29.04.1873 (Tunisia), leg. Abdul Kerim, det. \& donated by Raffaello Gestro.

Pheropsophus (Stenaptinus) javanus (Dejean, 1825)

Exotic collection (MGAB): 2 specs., Java (Indonesia), 1894, det. \& donated by Theodor Adensamer.

\section{ACKNOWLEDGEMENTS}

Our sincere thanks are due to Gabriela Cuzepan, curator at Natural History Museum Sibiu for the loan of specimens. The authors thank Dr. Alexandru Iftime for generating the distribution maps and George Năzăreanu for the photos. We are grateful to the reviewers for improving the paper.

\section{ASUPRA SPECIILOR DE BRACHININI (COLEOPTERA: CARABIDAE: BRACHININAE: BRACHININI) DIN CÂTEVA COLECŢII MUZEALE}

\section{REZUMAT}

În fauna României, se cunosc pe baza surselor bibliografice zece specii ce aparţin tribului Brachinini: Aptinus bombarda Illiger, Brachinus crepitans (Linnaeus), B. ejaculans Fischer von Waldheim, B. elegans Chaudoir, B. plagiatus Reiche, B. psophia Audinet-Serville, B. explodens Duftschmid, B. bayardi Dejean, B. bipustulatus Quensel și Mastax thermarum thermarum (Steven). $\mathrm{Au}$ fost studiate exemplarele aparţinând colecţiilor de coleoptere din două muzee de ştiințele naturii, cu cele mai mari colecţii: Muzeul National de Istorie Naturală "Grigore Antipa”, Bucureşti şi Muzeul National Brukenthal, Sibiu. În urma studiului exemplarelor din aceste colecţii, alte două specii de Brachinus se adaugă celor cunoscute: Brachinus brevicollis Motschulsky și B. nigricornis Gebler. Pe baza materialului studiat au fost realizate hărți de distribuție pentru toate cele 12 specii. Sunt prezentate fotografii ale habitusului celor două specii semnalate prima dată în fauna României şi sunt prezentate fotografii ale lobului median pentru şapte specii de Brachinus. Pe lângă exemplarele colectate din România, lucrarea prezintă şi exemplare/specii care provin din diverse alte ţări europene, dar şi din nordul Africii şi chiar Indonezia (Java).

\section{LITERATURE CITED}

BAEHR, M., 2004 - Brachininae. In: H. Freude, K. W. Harde, G. A. Lohse \& B. Klausnitzer, Die Käfer Mitteleuropas, 2, Adephaga 1: Carabidae (Laufkäfer). Spektrum-Verlag (Heidelberg/ Berlin), 2. Auflage.

BIELZ, E. A., 1887 - Die Erforschung der Käferfauna Siebenbürgens bis zum Schlusse des Jahres 1886. Verhandlungen und Mittheilungen des siebenbürgischen vereins für Naturwissenschaffen, 37: 3-90.

FIERA, C., D. PURICE, S. MAICAN, 2013 - The communities structure of invertebrate fauna from rape and alfalfa crops (Singureni, Giurgiu county, Romania). Cercetări Agronomice în Moldova, 46, 4 (156): 65-74.

FLECK, E., 1905 - Die Coleopteren Rumäniens. Bulletin de la Société des Sciences de BucarestRoumanie, 5-6: 402-465.

HŮRKA K., 1996 - Tribe Brachinini. In: Carabidae of the Czech and Slovak Republics. Illustrated key. Zlín, Kabourek. Pp. 134-139. (in Czech and English)

IENIŞTEA, M. AL., 1968 - Ord. Coleoptera (pars). Travaux du Muséum d'Histoire Naturelle "Grigore Antipa", 9: 97-117.

IENIŞTEA, M. AL., 1974 - Contributions à la connaissance des Coléoptères du Delta du Danube (Le "grind" Caraorman). Travaux du Muséum d'Histoire Naturelle "Grigore Antipa", 14: 239-249. 
IENIŞTEA, M. AL., 1975 - Coleoptera. In: Fauna. Grupul de cercetări complexe „Porţile de Fier”, seria monografică. Editura Academiei Republicii Socialiste România. pp. 193-210. (in Romanian)

JONESCO, C., 1911 - Contribution à la faune terrestre de Roumanie. Les Coléoptères des Environs de Jassy. Annales Scientifiques de l'Université de Jassy, 1-23.

MARCU, O., 1929 - Contribuţiuni la cunoaşterea Coleopterelor Olteniei. Buletinul Asociaţiei Naturaliştilor din Oltenia, 2-3: 1-20. (in Romanian)

MONTANDON, A. L., 1887 - Excursions en Dobroudja. Bulletin de la Societé Entomologique Scientific Angers (Nouvelle série), 14: 31-64.

MONTANDON, A. L., 1906 - Notes sur la faune entomologique de la Roumanie. (Coleoptera). Bulletin de la Société des Sciences de Bucarest - Roumanie, 16 (1-2): 30-80.

NEGRU, ŞT., 1957 - Contribuţiune la cunoaşterea faunei coleopterologice a Mangaliei şi împrejurimilor ei. Analele Universităţii C. I. Parhon-Bucureşti, Seria Ştiinţele Naturii. 16, 117-138. (in Romanian)

NEGRU, ŞT., A., ROŞCA, 1967 - Ord. Coleoptera. Travaux du Muséum d' Histoire Naturelle “Grigore Antipa", 7: 119-148.

NITZU, E., 1997 - Edaphicolous, endogeous and subterranean coleoptera from the movile karstic area (Southern Dobrogea, Romania). Travaux de l'Institut de Speologie "Émil Racovitza", 36: 73-98.

NITZU, E., 2001 - Edaphic and subterranean Coleoptera from the Dobrogean karstic area (Romania). A zoogeogarphic approach. Mitteilungen aus dem Hamburgischen Zoologischen Museum und Institut, 98: 131-169.

NITZU, E., 2007a - Ordinul Coleoptera, Familia Carabidae. In: O.T., Moldovan, M. Cimpean, D. Borda, S. Iepure, V. Ilie, (eds.), Lista faunistică a României (specii terestre şi de apă dulce)/ Checklist of Romanian fauna (terrestrial and freshwater species). Casa Cărţii de Ştiinţă, Cluj-Napoca, pp. 98-107.

NITZU, E., 2007b - Studii eco-faunistice asupra asociaţiilor de coleoptere edafice din zona Sic-Păstăraia (Câmpia Transilvaniei). Analele ICAS, 50:153-167. (in Romanian)

PETRI, K., 1912 - Siebenbürgens Käferfauna auf Grund ihrer Erforschung bis zu Jahre 1911. Siebenbürgischen Vereins für Naturwissenschaften zu Hermannstadt, 1-376.

PETRI, K., 1925-1926 - Ergänzungen und Berichtigungen zur Käferfauna Siebenbürgens 1912. Verhandlungen und Mitteilungen des Siebenbürgischen Vereins fur Naturwissenschaften zu Hermannstadt, 75-76: 1-42.

POPOVICI, L.-R., 1992 - Varietatea specifică a carabidelor din Delta Dunării - Rezervaţia Biosferei. Analele ştiinţifice ale Institutului Delta Dunării, Tulcea: 111-118. (in Romanian)

RUICĂNESCU, A., 1992 - Contribuţii la studiul comunităţilor de coleoptere din Cheile Turzii. Buletinul Informativ al Societății Lepidopterologice Române, 3 (4): 9-15. (in Romanian)

SĂVULESCU, N., A., POPESCU-GORJ, 1964 - Pădurile din sud-vestul Dobrogii, monumente puţin cunoscute ale naturii. Note. Ocrotirea naturii, 8 (2): 257-276. (in Romanian)

VARVARA, M., 2004 - Variation of the species diversity of Carabidae (Coleoptera, Carabidae) in two vegetal associations in the Bârnova Forest, Iaşi (east of Romania). Analele Ştiinţifice ale Universităţii "Al. I. Cuza" Iaşi, Biologie animală, 50: 117-139.

VARVARA, M., 2009 - Taxonomic diversity and the main ecological aspects of the coenosis of Carabidae in a wheat crop, Vaslui County. Buletin informativ al Societăţii lepidopterologice române, 20: 95-110.

VARVARA, M., F. ZUGRAVU, 2006 - Diversity and the main ecological requirements of the species of carabidae (Coleoptera, Carabidae) from Neamţ county. Analele Ştiinţifice ale Universităţii „Al. I. Cuza” Iaşi, seria Biologie animală, 52: 185-196.

*** http://carabidae.org/taxa/brachinini

Received: November 28, 2014

Accepted: February 6, 2015

\author{
Melania Stan \\ "Grigore Antipa" National Museum of Natural History \\ Sos. Kiseleff 1, 011341 Bucharest 2, Romania \\ e-mail: mstan@antipa.ro \\ Paul Gîdei \\ Str. Ștefan cel Mare, 611039, Roman, Neamț, Romania \\ e-mail: p_gidei@yahoo.com
}

